Article

\title{
Dynamic Changes in Serum Metabolomic Profiles of Growing Pigs Induced by Intravenous Infusion of Sodium Butyrate
}

\author{
Hongyu Wang ${ }^{1,2}$, Erdu Ren ${ }^{1,2}$, Xiaoe Xiang ${ }^{3}$, Yong Su ${ }^{1,2, *}$ and Weiyun Zhu ${ }^{1,2}$ \\ 1 Laboratory of Gastrointestinal Microbiology, Jiangsu Key Laboratory of Gastrointestinal Nutrition and \\ Animal Health, College of Animal Science and Technology, Nanjing Agricultural University, Nanjing 210095, \\ China; 2019205029@njau.edu.cn (H.W.); 2015105044@njau.edu.cn (E.R.); zhuweiyun@njau.edu.cn (W.Z.) \\ 2 National Center for International Research on Animal Gut Nutrition, Nanjing Agricultural University, \\ Nanjing 210095, China \\ 3 National Experimental Teaching Demonstration Center of Animal Science, Nanjing Agricultural University, \\ Nanjing 210095, China; xxxiang@njau.edu.cn \\ * Correspondence: yong.su@njau.edu.cn; Tel.: +86-25-84395523
}

Received: 4 November 2019; Accepted: 29 December 2019; Published: 1 January 2020

\begin{abstract}
This study aimed to explore the dynamic changes in metabolite profiles and metabolism pathways in the serum of growing pigs by intravenous infusion of sodium butyrate (SB). Fourteen crossbred growing barrows $(\mathrm{BW}=23.70 \pm 1.29 \mathrm{~kg}$ ) fitted with jugular cannula were randomly allocated to the SB and control (Con) groups, each group consisted of seven replicates (pens), with one pig per pen. At 9:00 of each day during the experimental period, pigs in the SB group were infused with $10 \mathrm{~mL}$ of $\mathrm{SB}\left(200 \mathrm{mmol} / \mathrm{L}, \mathrm{pH} 7.4,37^{\circ} \mathrm{C}\right)$ via precaval vein, while the Con group was treated with the same volume of physiological saline. On day 4 , the blood of each pig was collected at $0,30,60$, and $120 \mathrm{~min}$ after the intravenous infusion. Metabolites in the serum were detected by gas chromatograph-mass spectrometry analysis. Pathway analysis of metabolomic profiles showed that the differential metabolites mainly enriched in amino acid metabolism, lipid-related metabolism, and the tricarboxylic acid (TCA) cycle. More importantly, the relative concentrations of all eight essential amino acids, five non-essential amino acids, and two amino acid derivatives were decreased by the parenteral SB. In addition, SB significantly increased the relative concentrations of eicosanoic acid and octadecanoic acid and decreased the relative concentration of glycerol-3-phosphate at $0 \mathrm{~min}$ (three days after intravenous infusion of SB), which suggests that parenteral SB may increase stearates mobilization and decrease the biosynthesis of stearates. In conclusion, intravenous infusion of SB may induce more amino acids to synthesize proteins and affect fat metabolism through increasing fat mobilization and decreasing the biosynthesis of stearates. However, a further study is needed to understand the mechanism of extensive metabolic pathway changes induced by parenteral SB.
\end{abstract}

Keywords: sodium butyrate; serum metabolomic; intravenous infusion; growing pig

\section{Introduction}

Butyrate is one of the short-chain fatty acids (SCFA) with four-carbon, which is mainly produced by microbial fermentation [1,2]. It has received particular attention for its beneficial effects on improving immunity and intestinal health $[3,4]$, regulating intestinal microecology $[5,6]$, inhibiting bacterial growth [7], and promoting growth [8]. Therefore, butyrate preparations (such as sodium butyrate (SB) and butyrate glycerides) have been widely used in pig $[9,10]$, poultry $[11,12]$ and aquatic $[13,14]$ feed. It is well documented that butyrate is the major energy source for colonocytes [15]. As regulating molecule, butyrate exerts an effect on energy metabolism based on G-protein coupled 
receptors (GPCR) [16], and butyrate is a typical histone deacetylase (HDAC) inhibitor which mediates physiological function by inducing histone hyperacetylation to improve genetic transcription [17]. Meanwhile, it was proven that butyrate could increase the muscle fiber cross-sectional area, prevent intramuscular fat accumulation, and improve glucose metabolism in aging mice [18]. Overall, butyrate plays an important role in maintaining nutrient metabolism and the health of an animal.

Interestingly, a study has shown that $90 \%$ of the butyrate produced in the gut was used by colon cells [19], with only a small portion going into the bloodstream. Even so, butyrate still plays a big role in body metabolim. Several reports in vitro showed that butyrate could stimulate porcine adipocyte differentiation [20], induce metabolic adaptations [21], and stimulate leptin production in adipocytes [22]. Moreover, researches have found that intravenous injection of SB could increase the secretion of amylase [23], improve structural aspects of intestinal adaptations by increasing proliferation and decreasing apoptosis [24], change the gene expression of the intestine [25] and the liver [26], and remold the composition of intestinal microecology [27] in animal models with intestine injury. Our previous study [26] showed that the infusion of butyrate changed the metabolite profile of the liver in a healthy growing pig model. However, limited information is available on the impact of parenteral butyrate on the profile of serum metabolites and its dynamics changes in pigs.

In this study, we hypothesized that the parenteral butyrate may have extensive effects on amino acid metabolism, fat metabolism, and energy metabolism accompanied with the dynamic changes of metabolite concentrations participated in these pathways. Therefore, the purpose of this study was to explore the dynamic change of the metabolite profile and metabolism pathway in the serum of growing pigs by intravenous butyrate through gas chromatograph-mass spectrometry (GC-MS) analysis.

\section{Results}

\subsection{Effects of Intravenous Infusion of SB on Serum Biochemical Parameters}

Serum metabolite concentrations of pigs in control (Con) and SB groups are shown in Table 1. The infusion of SB increased the concentration of high-density lipoprotein-cholesterol (HDL-C) $(p=0.005)$, while there was no difference in the concentrations of low-density lipoprotein-cholesterol (LDL-C), glucose, and triglyceride $(p>0.05)$ between two groups (Table 1). The main effect of time and interaction effect was not detected between groups at $0,30,60$, and $120 \mathrm{~min}$.

Table 1. Serum metabolite concentrations of pigs in the control (Con) and sodium butyrate (SB) groups at $0,30,60,120 \mathrm{~min}$ after intravenous infusion $(n=7)^{1}$.

\begin{tabular}{|c|c|c|c|c|c|c|c|c|c|c|c|c|}
\hline \multirow{2}{*}{ Item } & \multicolumn{4}{|c|}{ Con } & \multicolumn{4}{|c|}{ SB } & \multirow{2}{*}{ SEM } & \multicolumn{3}{|c|}{$p$ Value } \\
\hline & 0 & 30 & 60 & 120 & 0 & 30 & 60 & 120 & & Time & SB & Interaction \\
\hline Total protein & 49.83 & 53.17 & 50.13 & 50.79 & 54.43 & 51.5 & 51.49 & 53.03 & 2.14 & 0.787 & 0.161 & 0.293 \\
\hline Cholesterol & 1.94 & 1.93 & 1.91 & 1.96 & 2.15 & 2.01 & 2.04 & 2.09 & 0.19 & 0.930 & 0.176 & 0.973 \\
\hline HDL-C & 0.90 & 0.94 & 0.93 & 0.95 & 1.13 & 1.05 & 1.02 & 1.06 & 0.08 & 0.939 & 0.005 & 0.699 \\
\hline LDL-C & 1.14 & 1.19 & 1.14 & 1.17 & 1.22 & 1.16 & 1.13 & 1.19 & 0.13 & 0.969 & 0.972 & 0.927 \\
\hline
\end{tabular}

${ }^{1}$ HDL-C = high-density lipoprotein-cholesterol; LDL-C = low-density lipoprotein-cholesterol; SEM = Standard Error of Mean.

\subsection{Global Overview of the Serum Metabolomic Profile}

The standard deviations of internal standards in Quality Control (QC) were both below 1\% (Supplementary Material, Table S1). With the GC-MS method, 232 peaks were obtained. A total of 75 metabolites including fatty acid, amino acid, organic acid, saccharides, alcohols, amine, pyrimidone, and purines (Supplementary Material, Table S2) were identified in the serum of pigs in both Con and SB groups.

Time-series analysis combined with one experimental factor method was used to explore the main effects of time and SB infusion on the serum metabolites and their interaction effect. These results 
(Supplementary Material, Table S3) indicated that 48 metabolites were affected by the infusion of SB $(p<0.05)$ and 19 metabolites were changed with time $(p<0.05)$. Among them, only 11 metabolites had an interaction effect $(p<0.05)$. Pathway enrichment analysis showed that the influenced metabolism pathway mainly enriched in alanine, aspartate and glutamate metabolism, arginine and proline metabolism, glycine, serine and threonine metabolism, butanoate metabolism, glycerophospholipid metabolism, and the tricarboxylic acid (TCA) cycle (Figure 1).

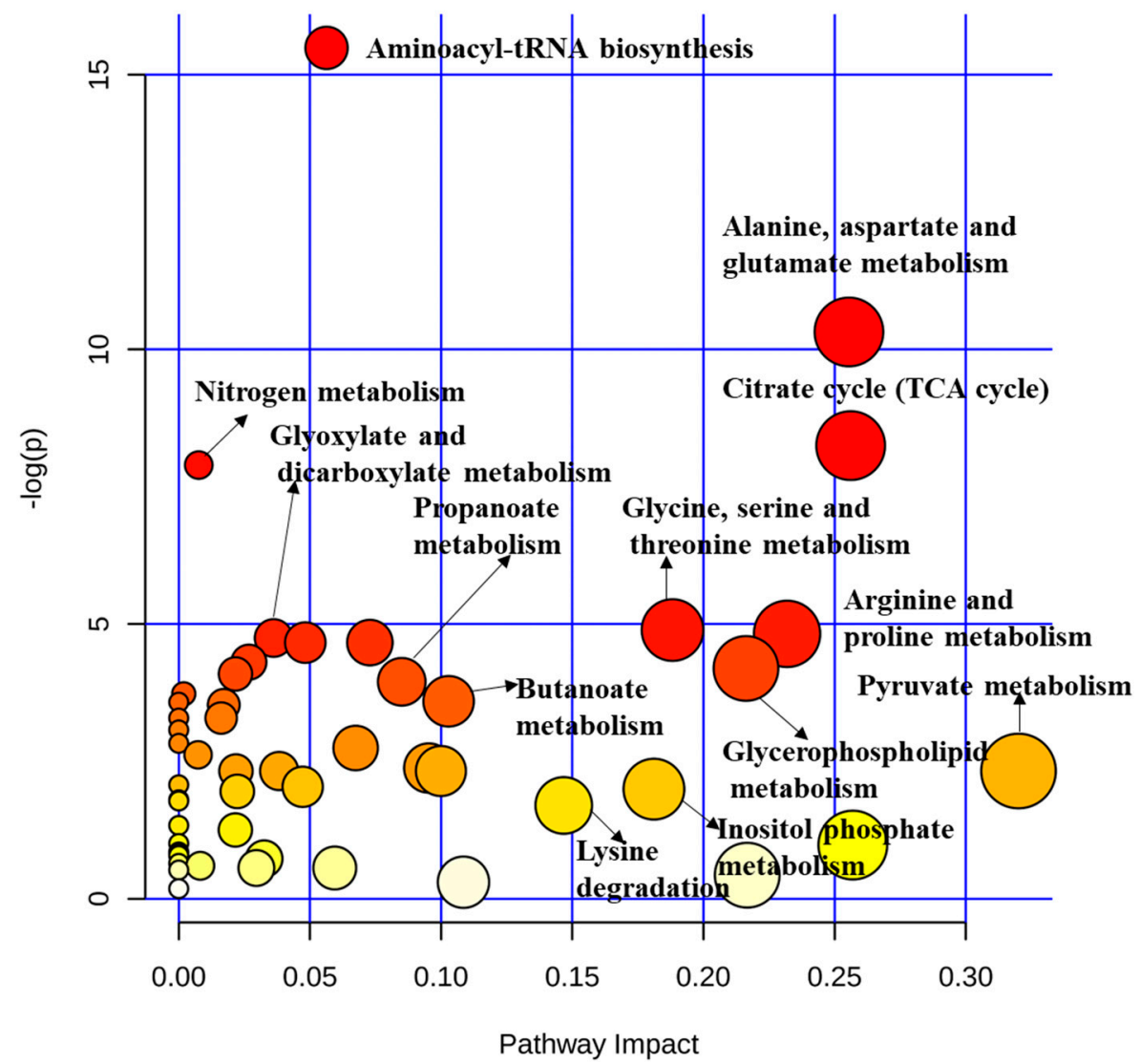

Figure 1. Significantly changed pathways of serum metabolites in pigs affected by the infusion of sodium butyrate (SB) from a whole period perspective. Here, the $x$-axis represents the pathway impact and the $y$-axis represents the pathway enrichment. Each node marks a pathway, with larger sizes and darker colors representing higher pathway enrichment and higher pathway impact values.

Venn digrams of differential metabolites and enriched metabolic pathways at different timepoints showed that two differential metabolites (methionine and tyrosine) and one metabolic pathway (phenylalanine tyrosine and tryptophan biosynthesis) were all influenced at four timepoints (Figure 2).

\subsection{Effects of Intravenous Infusion of SB on Serum Metabolomics of Growing Pigs}

As shown in Figures 3 and 4, the score plots of both principal component analysis (PCA) and partial least squares discriminant analysis (PLS-DA) distinguished the SB and Con groups at 0, 30, 60, and $120 \mathrm{~min}$ after SB infusion. 


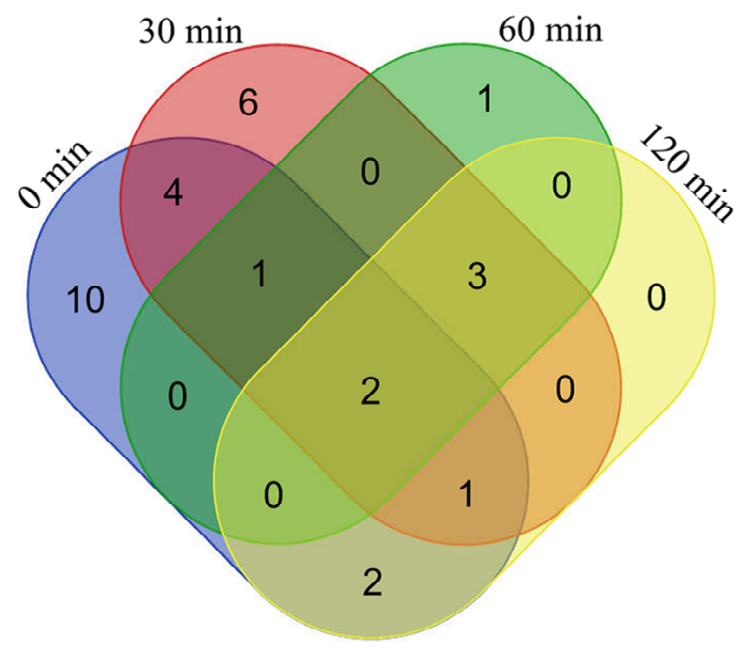

Differential metabolites

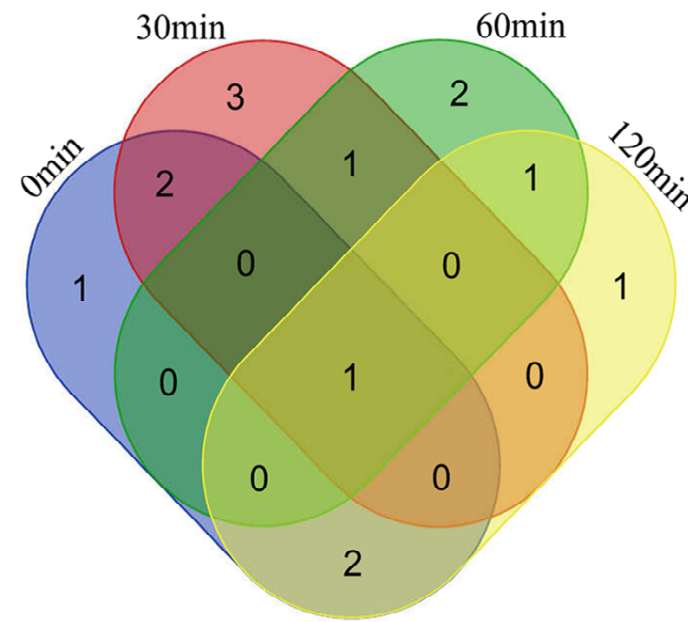

Metabolic pathway

Figure 2. Venn diagrams of differential metabolites and enriched metabolic pathways at the timepoints of $0,30,60$, and $120 \mathrm{~min}$.
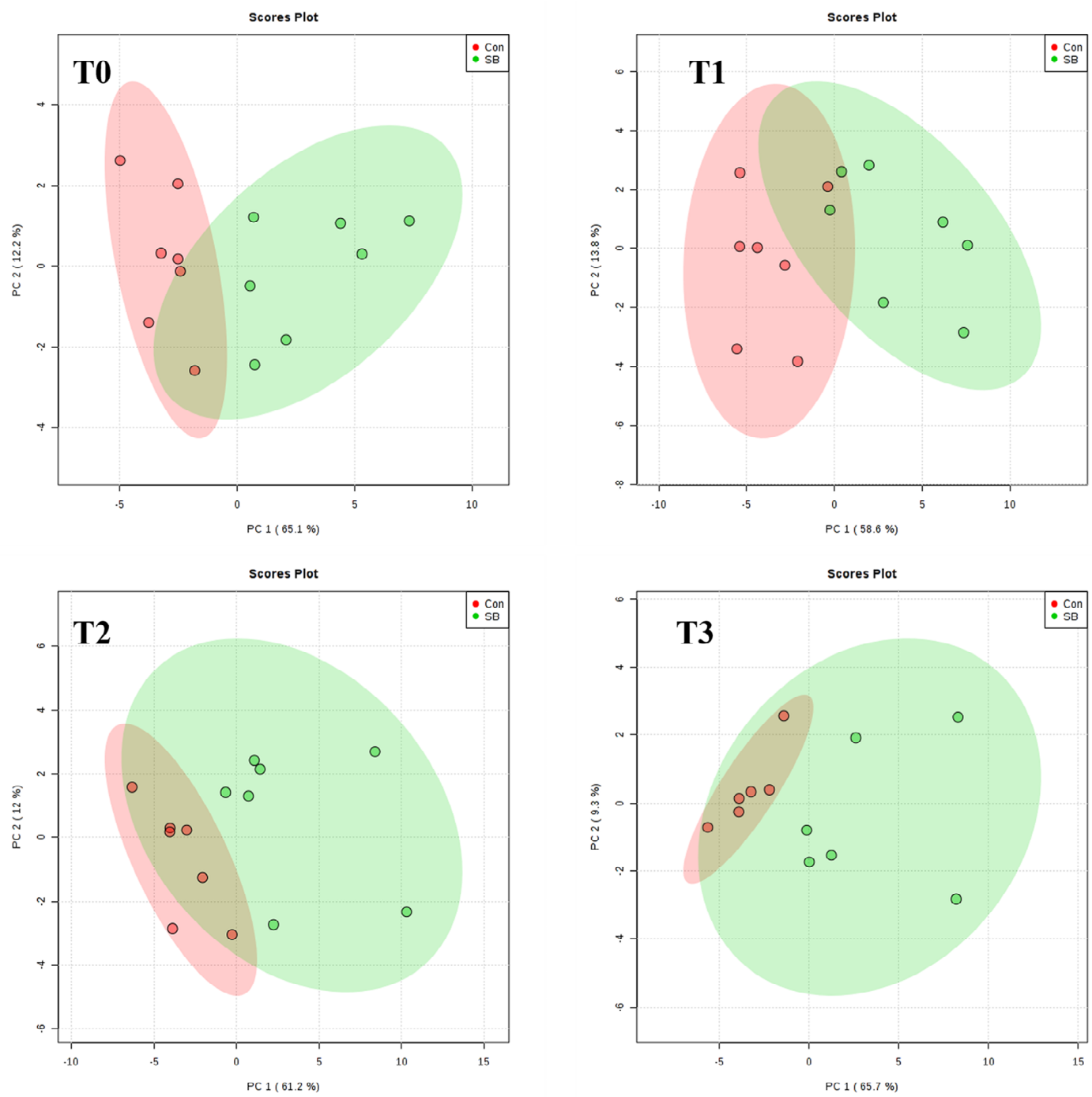

Figure 3. Principal component analysis (PCA) score plot of metabolites of pigs in the control (Con) and sodium butyrate (SB) groups at T0 ( $0 \mathrm{~min}, n=7), \mathbf{T} 1$ (30 $\mathrm{min}, n=7), \mathbf{T} 2(60 \mathrm{~min}, n=7)$, and T3 (120 $\min , n=6$ ) after intravenous infusion. 

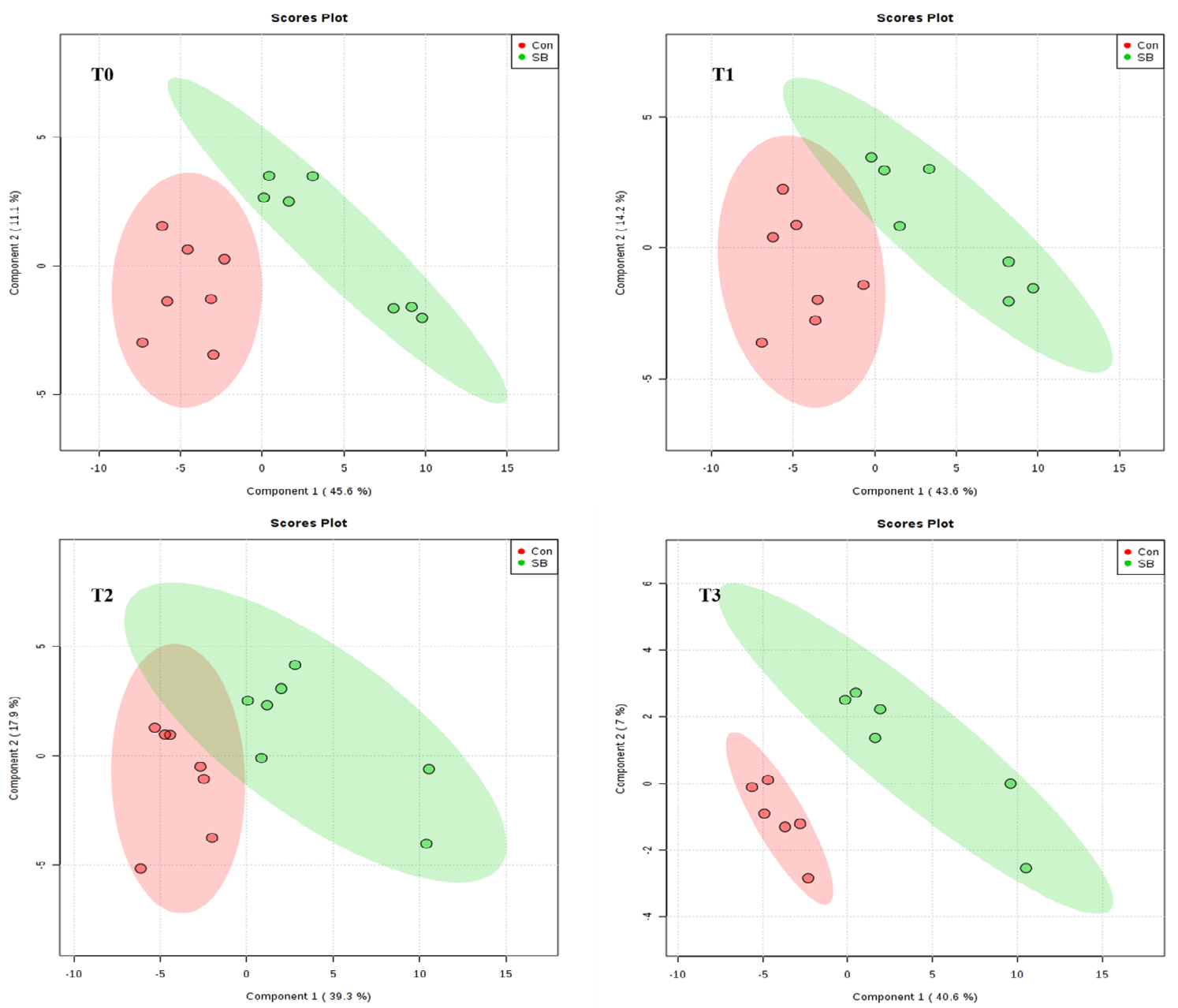

Figure 4. Partial least squares discriminant analysis (PLS-DA) score plot of metabolites of pigs in the control (Con) and sodium butyrate (SB) groups at T0 ( $0 \mathrm{~min}, n=7)$, T1 (30 $\mathrm{min}, n=7)$, T2 (60 $\mathrm{min}, n=7)$, and T3 (120 $\mathrm{min}, n=6)$ after intravenous infusion. Component $1=$ the first principal; Component 2 $=$ the second principal. The explained variances of the first two components are shown in brackets, respectively. The ellipse represents the $95 \%$ confidence interval of each group.

At 0 min, just before intravenous infusion of day 4 and actually three days after the first intravenous infusion, 20 metabolites were identified through combination methods of univariate and multivariate analysis (Supplementary Materials, Table S4). Pathway enriched analysis results (Figure 5, T0) indicated that these 20 differential metabolites mainly enriched in alanine, aspartate and glutamate metabolism, D-glutamine and D-glutamate metabolism, phenylalanine metabolism, arginine biosynthesis, phenylalanine, tyrosine and tryptophan biosynthesis, and TCA cycle.

At $30 \mathrm{~min}, 17$ differential metabolites were identified compared with the Con group (Supplementary Materials, Table S5). These differential metabolites mainly enriched in alanine, aspartate and glutamate metabolism, arginine and proline metabolism, beta-alanine metabolism, glycine, serine and threonine metabolism, phenylalanine metabolism, D-Glutamine and D-glutamate metabolism, arginine biosynthesis, phenylalanine, tyrosine and tryptophan biosynthesis, and glycerolipid metabolism (Figure 5, T1).

At $60 \mathrm{~min}$, seven differential metabolites were identified respectively of the SB group compared with the Con group (Supplementary Materials, Table S6). These differential metabolites mainly enriched in phenylalanine, tyrosine and tryptophan biosynthesis, cysteine and methionine metabolism, tryptophan metabolism, and tyrosine metabolism at $60 \mathrm{~min}$ (Figure 5, T2). 
At $120 \mathrm{~min}, 28$ differential metabolites were identified of the SB group compared with the Con group (Supplementary Materials, Table S7). These differential metabolites mainly enriched in alanine, aspartate and glutamate metabolism, phenylalanine, tyrosine and tryptophan biosynthesis, cysteine and methionine metabolism, the TCA cycle, and glyoxylate and dicarboxylate metabolism (Figure 5, T3).
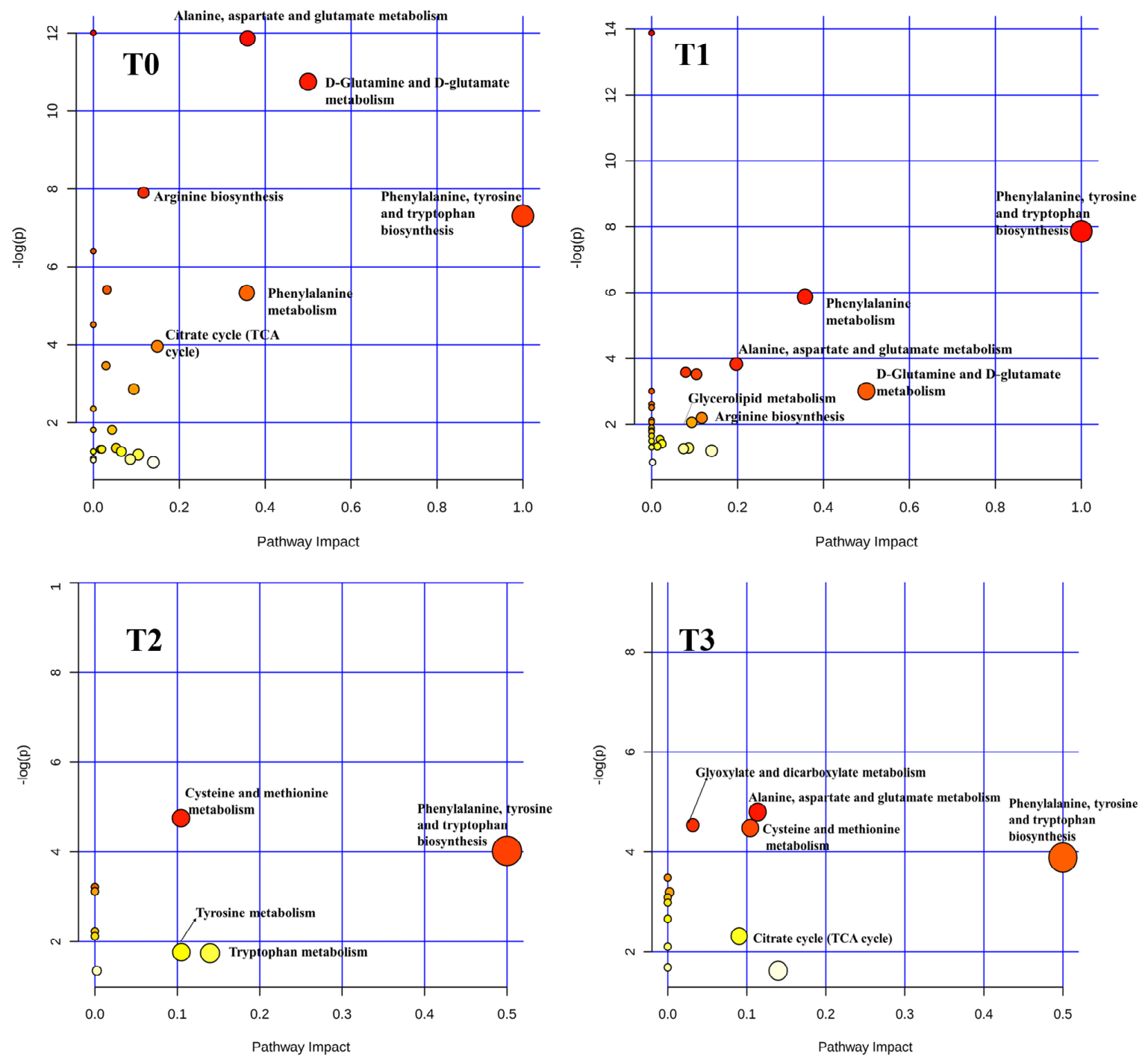

Figure 5. Significantly changed pathways of pigs in the sodium butyrate (SB) group compared with the control (Con) group at T0 (0 min, $n=7)$, T1 (30 $\mathrm{min}, n=7)$, T2 $(60 \mathrm{~min}, n=7)$, and T3 (120 $\mathrm{min}$, $n=6)$ after intravenous infusion. Here, the $x$-axis marks the pathway impact and the $y$-axis represents the pathway enrichment. Each node marks a pathway, with larger sizes and darker colors representing higher pathway enrichment and higher pathway impact values.

\subsection{Effects of Intravenous Infusion of SB on the Dynamic Changes of Differential Metabolites}

The dynamic changes of relative concentrations of mainly differential metabolites are shown in Figures 6-8. In the current study, seventeen of the 20 amino acids (except histidine, arginine, and aspartic acid) were detected in the serum of growing pigs from both SB and Con groups (Supplementary Materials, Table S2). The intravenous infusion of SB significantly decreased the relative concentrations of all eight essential amino acid detected in the serum and included lysine (30,60, and $120 \mathrm{~min}, p<0.05)$, methionine $(0,30,60$, and $120 \mathrm{~min}, p<0.05,0.01,0.01$, and 0.05 , respectively), phenylalanine ( 0 and 30 $\min , p<0.05)$, leucine $(0,30$, and $60 \mathrm{~min}, p<0.05,0.01$, and 0.01 , respectively), isoleucine $(0,30$, and 
$60 \mathrm{~min}, p<0.01,0.001$, and 0.001, respectively), threonine $(0,30,60$, and $120 \mathrm{~min}, p<0.05,0.01,0.01$, and 0.05 , respectively), valine $(30,60$, and $120 \mathrm{~min}, p<0.01)$, and tryptophan $(0$ and $30 \mathrm{~min}, p<0.05)$, five non-essential amino acids, proline $(0,30$, and $60 \mathrm{~min}, p<0.05,0.01$, and 0.01 , respectively), serine $(0,30$, and $60 \mathrm{~min}, p<0.05,0.01$, and 0.05 , respectively), tyrosine $(0,30,60$, and $120 \mathrm{~min}, p<0.05)$, cysteine (30,60, and $120 \mathrm{~min}, p<0.001,0.001$, and 0.01 , respectively), and glutamine (0, 30, and 120 $\min , p<0.01,0.05$, and 0.05 , respectively), and two amino acid derivatives, $\beta$-alanine $(0,30$, and 120 $\min , p<0.01,0.05$, and 0.05 , respectively) and ornithine $(0,30,60$, and $120 \mathrm{~min}, p<0.01,0.01,0.05$, and 0.05 , respectively).

\section{Essential amino acid}
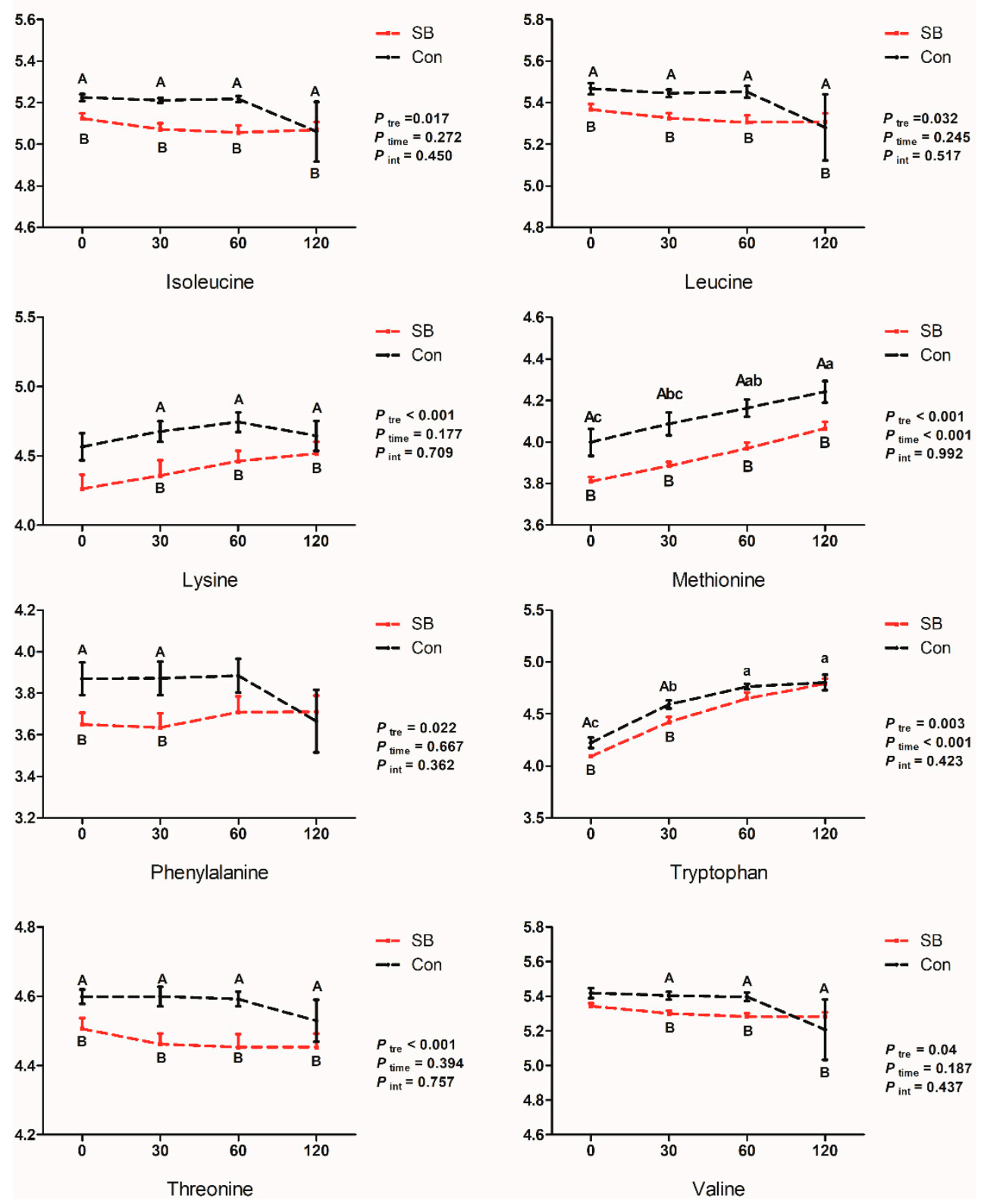

Figure 6. Dynamic changes of the relative concentrations of essential amino acids. $X$-axis presents the relative concentrates of corresponding metabolites with Log processing of the peak area. Differences were analyzed by two-way ANOVA using PROC MIXED with time and SB treatment as the main factors followed by post-hoc analysis in SAS. Ptre, Ptime, Pint represents the significances of SB treatment, time, and interaction effects, respectively. Superscript with A or B means significant difference between control group (Con) and sodium butyrate group (SB). Superscript with a, b, and c means significant difference over time. 

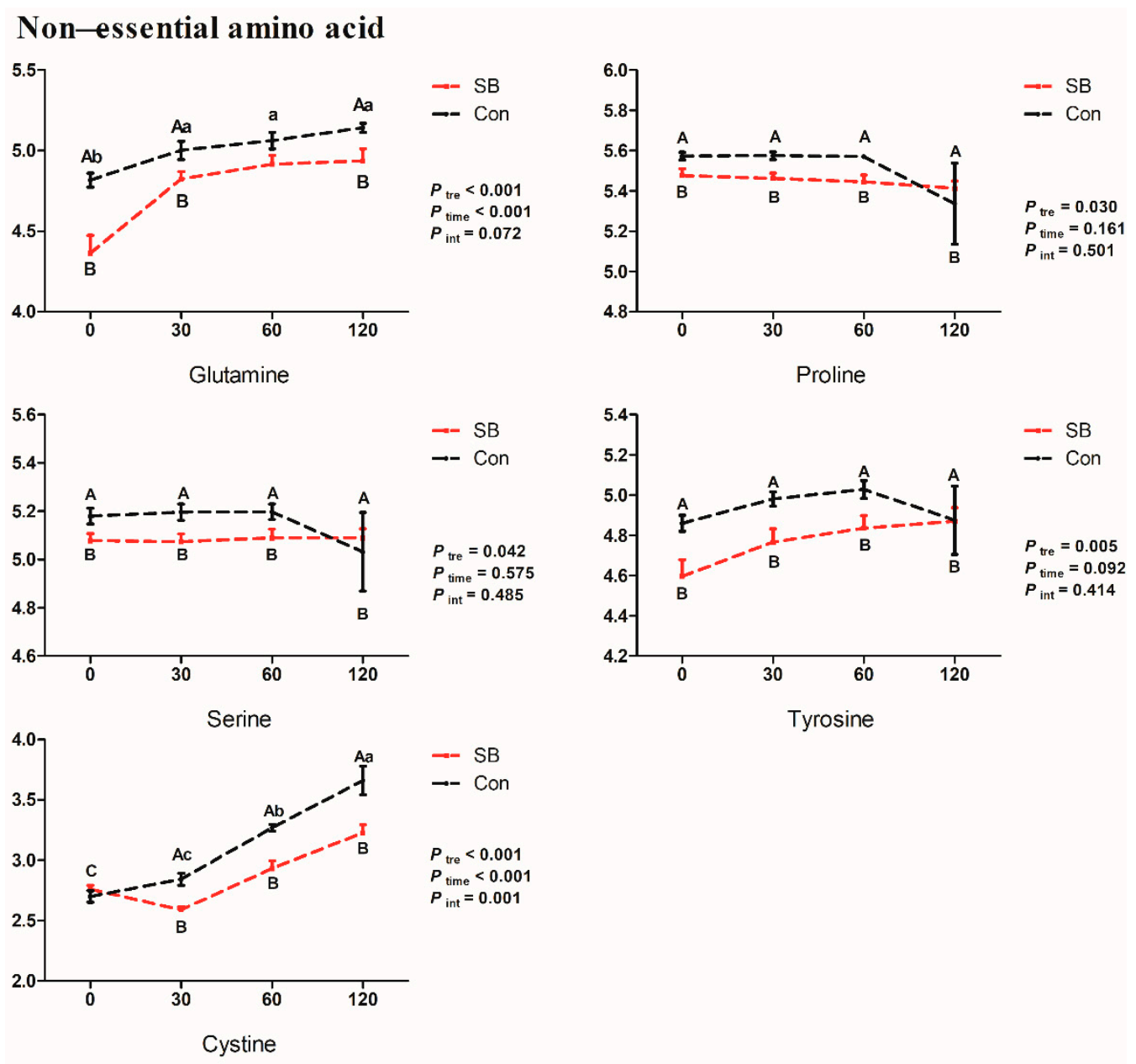

Amino acid derivatives
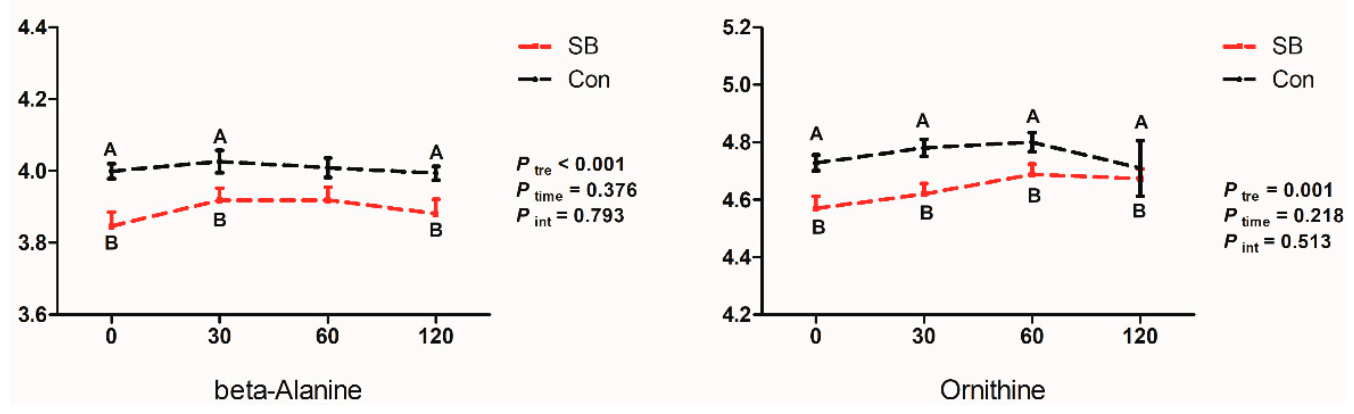

Figure 7. Dynamic changes of the relative concentrations of non-essential amino acids and derivatives. $\mathrm{X}$-axis presents the relative concentrates of corresponding metabolites with Log processing of the peak area. Differences were analyzed by two-way ANOVA using PROC MIXED with time and SB treatment as the main factors followed by post-hoc analysis in SAS. Ptre, Ptime, Pint represents the significances of SB treatment, time, and interaction effects, respectively. Superscript with A or B means significant difference between control group (Con) and sodium butyrate group (SB). Superscript with a, b, and c means significant difference over time.

The intravenous infusion of SB significantly increased the relative concentrations of eicosanoic acid $(p<0.001)$ and octadecanoic acid $(p<0.001)$ at $0 \mathrm{~min}$ while no difference was detected at 30,60 , and $120 \mathrm{~min}(p>0.05)$. Meanwhile, the infusion of SB significantly decreased the relative concentrations of arachidonic acid at 0,30 , and $60 \mathrm{~min}(p<0.05)$ and nonanoic acid at $30 \mathrm{~min}(p<0.05)$. Moreover, the relative concentrations of glycerol-3-phosphate $(0 \mathrm{~min}, p<0.01)$ and glyceric acid $(0,30$, and $60 \mathrm{~min}, p<0.05,0.01$, and 0.05 , respectively) were significantly decreased. Moreover, the relative 
concentrations of $\alpha$-ketoglutaric acid ( $0 \mathrm{~min}, p<0.001)$, citric acid (0, 60, and $120 \mathrm{~min}, p<0.01,0.05$, and 0.05 , respectively), and oxalic acid (0,30, and $120 \mathrm{~min}, p<0.001,0.05$, and 0.05 , respectively) were significantly decreased by the infusion of SB.
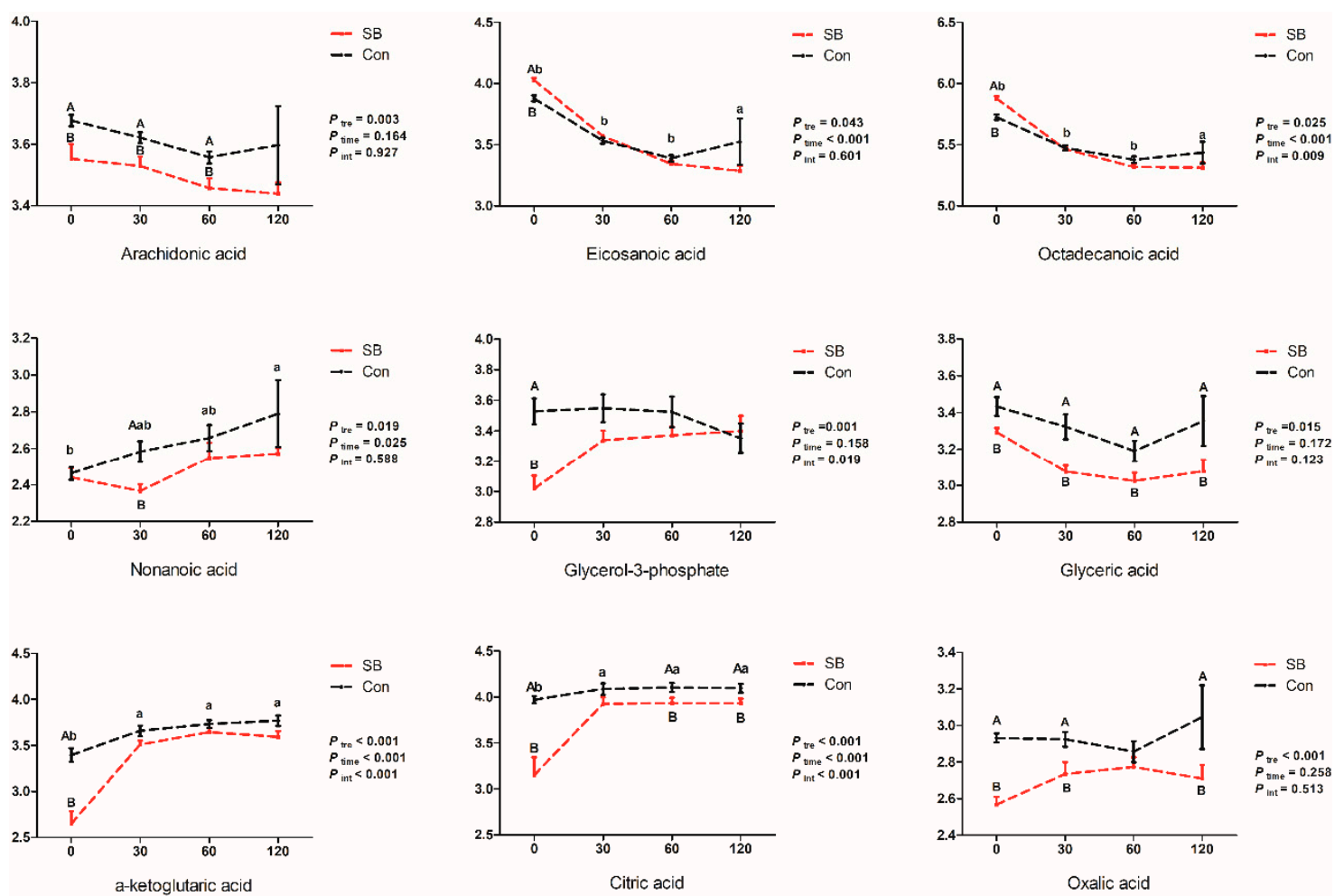

Figure 8. Dynamic changes of the relative concentration of fatty acids and organic acids. X-axis presents the relative concentrations of corresponding metabolites with Log processing of the peak area. Differences were analyzed by two-way ANOVA using PROC MIXED with time and SB treatment as the main factors followed by post-hoc analysis in SAS. Ptre, Ptime, Pint represents the significances of SB treatment, time, and interaction effects, respectively. Superscript with A or B means significant difference between control group (Con) and sodium butyrate group (SB). Superscript with a, b, and c means significant difference over time.

\section{Discussion}

The effects of SB on the health and metabolism of pigs have been widely confirmed [28-31]. Likewise, the results in this experiment proved that intravenous infusion of SB had comparatively extensive effects on the metabolism of pigs, mainly focusing on amino acid metabolism, fatty acid metabolism, and the TCA cycle, especially on amino acid metabolism.

\subsection{Effects of Intravenous Infusion of SB on Amino Acid Metabolism of Growing Pigs}

While intensive studies mainly focused on the impact of butyrate on glycolipid metabolism, little is known on amino acid metabolism. In this study, it is surprising that the parenteral SB had a profound effect on amino acid metabolism. These metabolites were mainly enriched in alanine, aspartate and glutamate metabolism, phenylalanine, tyrosine and tryptophan biosynthesis, cysteine and methionine metabolism, phenylalanine metabolism, D-Glutamine and D-glutamate metabolism, and arginine biosynthesis. Consistently, Yu et al. (2017) [32] reported that oval butyrate changed beta-alanine metabolism, alanine metabolism, and cysteine metabolism in $\mathrm{d} 7$ and glycine, serine and threonine metabolism in $\mathrm{d} 21$ in the liver of neonatal piglets. In addition, previous research proved that intravenous infusion of SB could change the pathway of glycine, serine and threonine metabolism, beta-Alanine metabolism, and methionine metabolism in the liver of growing piglets [26]. These results 
suggested that whether oral or intravenous infusion, SB could affect amino acid metabolism of pigs (newborn or growing pigs). The study herein demonstrated a more extensive role of parenteral SB in amino acid metabolism. Surprisingly, the relative concentrations of all detected differential metabolites including amino acids, fatty acids, and carbonhydrates were decreased after intravenous infusion of SB. Thus, we may speculate that parenteral SB enhanced the metabolism of nutrients (especially amino acids) in the body two hours after infusion. However, it is not clear whether this impact can last all day long. In addition, as a short-term experiment, no difference in the growth performance was observed between two groups. In the current study, there is insufficient knowledge to explain the molecular mechanism of the effects of parenteral SB on amino acid metabolism.

\subsection{Effects of Intravenous Infusion of SB on Fat Metabolism of Growing Pigs}

HDL-C is synthesized by the liver and can transport cholesterol from the extrahepatic tissues to the liver for metabolism. It has a negative correlation with the incidence of coronary heart disease, atherosclerosis, and other diseases [33]. In accordance with the research results of Jian et al. (2016) in mice [34], the results herein suggested that the infusion of SB increased serum HDL-C concentration. However, some previous studies showed that butyrate had no effect or reduced HDL-C concentration in serum. The reason for the inconsistent results may be the difference in sampling time and experimental animals, even a difference in the dose of SB [35-37]. As a crucial substance in fat metabolism, HDL-C is highly correlated to lipid metabolism pathway [38]. Specifically, our results showed that parenteral SB increased the relative concentration of long-chain saturated fatty acids eicosanoic and octadecanoic acid and decreased the relative concentration of glycerol-3-phosphate, which indicates that adipokinetic action is increased and fat synthesis is decreased by intravenous infusion of SB. Previous studies also proved that butyrate alleviated high fat diet-induced fat accumulation [34] and affected lipid metabolism in many ways [39-41]. In addition, pathway enrichment analysis results showed that arachidonic acid metabolism, glyoxylate and dicarboxylate metabolism, and glycerophospholipid metabolism were affected by the intravenous infusion of SB. In line with the result of this study, our previous study also showed that lipid metabolism-related genes and liver lipid metabolism profiles were regulated by the infusion of SB [26].

\subsection{Effects of Intravenous Infusion of SB on the TCA Cycle of Growing Pigs}

TCA cycle is the most effective way for animals to obtain energy, and it is the hub of the metabolism of sugars, lipids, and amino acids [42,43]. In line with the results of Yu et al. (2016) [32], the intervention of SB could affect the TCA cycle. The relative concentrations of key substances in the TCA cycle of citric acid, a-ketoglutaric acid, and oxalic acid were all decreased, which may signify that parenteral SB has changed the metabolism of TCA cycle. However, these effects were not consistent at different timepoints. Since the molecular mechanism of the effect of parenteral SB on TCA is still unclear, we deduced that butyrate may exert its effects on the TCA cycle in the following ways. Firstly, as free fatty acid butyrate can be oxidized through $\beta$-oxidation pathway in which a fatty acid is broken down to an acetyl-CoA molecule via a number of intermediate steps [44]. Acetyl-CoA can entirely oxidize into $\mathrm{CO} 2$ to generate ATP or further participate in other metabolic pathways, such as long-chain fatty acid biosynthesis, cholesterol synthesis, and ketone body formation. Secondly, SB can indirectly affect the TCA cycle by affecting fatty acid metabolism and amino acid metabolism, which was firmly confirmed in this study. Thirdly, studies showed that butyrate can influence energy metabolism through stimulating mitochondrial function [34] and affecting GPCR [16].

\section{Material and Methods}

\subsection{Ethics Statement}

This study was approved by the Nanjing Agricultural University Animal Care and Use Committee (Nanjing, Jiangsu Province, China) (SYXK2017-0027). All surgical and animal care procedures in the 
experiment were operated according to the standard of Experimental Animal Care and Use Guidelines of China (EACUGC2018-01). The pigs had ad libitum access to food and water and were raised under suitable circumstances.

\subsection{Animals, Housing, and Experimental Design}

Fourteen crossbred growing barrows (Duroc $\times$ Landrace $\times$ Large White) were used in this experiment. Pigs were surgically fitted with a medical polyethylene cannula (inside diameter $2 \mathrm{~mm}$ and outside diameter $3 \mathrm{~mm}$ ) via an internal jugular vein for the infusion of SB. The experimental period was $4 \mathrm{~d}$ after a one-week recovery. After the recovery period, pigs (barrows $(\mathrm{BW})=23.70 \pm 1.29 \mathrm{~kg}$ ) were then randomly allocated into the SB infusion (SB) group and control (Con) group with seven replicates in each group and one pig per pen. At 9:00 of each day during the experimental period, pigs in the SB group were infused with $10 \mathrm{~mL}$ of SB $\left(200 \mathrm{mmol} / \mathrm{L}, \mathrm{PH} 7.4,37^{\circ} \mathrm{C}\right)$ via internal jugular vein, while the Con group was treated with the same dose of physiological saline. The dosage was chosen according to previous studies in pigs including butyrate through parenteral nutrition [24] or through oral administration [6]. During the whole experimental period and recovery period, all pigs were housed into $1.0 \times 1.2 \mathrm{~m}$ individual pens and fed with commercial feeds with metabolizable energy of $3.19 \mathrm{Mcal} / \mathrm{kg}$ and crude protein of $16.8 \%$ in as-fed basis. The pigs had ad libitum access to food and water throughout the experimental period.

\subsection{Sampling}

Piglets were fasted overnight on day 4 , blood samples of each pig were collected from the precaval vein at 0 (before infusion), 30, 60, and $120 \mathrm{~min}$ after infusion, respectively. After sampling, blood samples were centrifuged for $10 \mathrm{~min}$ at room temperature, the supernatant samples (serum) were collected and stored at $-80^{\circ} \mathrm{C}$ for the biochemical indexes and metabolites analysis.

\subsection{Serum Biochemical Analysis}

The concentrations of HDL-C, LDL-C, cholesterol, glucose, and triglyceride in the serum of piglets were determined by an Olympus AU400 Biochemical Analyzer (Tokyo, Japan) following the instrument's specifications.

\subsection{Gas Chromatograph-Mass Spectrometry Based Metabolites Profiling}

\subsubsection{Serum Samples Preparation and GC-MS Analysis}

The serum samples were pretreated, extracted, and derivatized as previously reported [26]. Briefly, $100 \mu \mathrm{L}$ of each sample was mixed with $400 \mu \mathrm{L}$ methanol containing 2-chlorobenzene alanine $(0.2 \mathrm{mg} / \mathrm{mL})$ and heptadecanoic acid $(0.2 \mathrm{mg} / \mathrm{mL})$, and vortex-mixed for $1 \mathrm{~min}$. Next, the samples were centrifuged at $12,000 \mathrm{rpm}\left(4^{\circ} \mathrm{C}\right)$ for $10 \mathrm{~min}$, and all serum was dried in a Vacuum centrifugal concentrator (LNG-T83, Huamei, Taicang, China). The dried analytes were mixed with a $60 \mu \mathrm{L}$ aliquot of methoxyamine pyridine solution $(15 \mathrm{mg} / \mathrm{mL})$, then vortex-mixed for $30 \mathrm{~s}$ and maintained for $2 \mathrm{~h}$ at $37^{\circ} \mathrm{C}$ for methoxidation. Then, $60 \mu \mathrm{L}$ BSTFA reagent was added (containing $1 \%$ trimethylchlorosilane), maintained for $90 \mathrm{~min}$ at $37^{\circ} \mathrm{C}$, and then the samples were centrifuged at $12,000 \mathrm{rpm}\left(4^{\circ} \mathrm{C}\right)$ for $10 \mathrm{~min}$, and the supernatants were added to the test bottles.

The derivatized samples $(1.0 \mu \mathrm{L})$ were injected into an Agilent 7890A GC system equipped with a fused-silica capillary column (30 $\mathrm{m} \times 0.25 \mathrm{~mm}$ i.d.) and chemically bonded with $0.25 \mu \mathrm{m}$ DB-5 stationary phase (J\&W Scientific, Folsom, CA, USA) by an Agilent 7683 Series autosampler (Agilent Technologies, Atlanta, GA, USA). The inlet temperature was set at $280{ }^{\circ} \mathrm{C}$. Helium was used as the carrier gas with a constant flow rate of $1.0 \mathrm{~mL} / \mathrm{min}$ through the column. The column temperature was initially maintained at $70{ }^{\circ} \mathrm{C}$ for $2 \mathrm{~min}$ and then increased at a rate of $10^{\circ} \mathrm{C} / \mathrm{min}$ from $70{ }^{\circ} \mathrm{C}$ to $300{ }^{\circ} \mathrm{C}$, where it was held for $5 \mathrm{~min}$. The column effluent was introduced into the ion source (maintained at $250{ }^{\circ} \mathrm{C}$ ) of a Pegasus III mass spectrometer (Leco, St. Joseph, MI, USA) through a transfer line, with the 
temperature set at $250^{\circ} \mathrm{C}$. Mass fragmentation was generated with an electron beam at $70 \mathrm{eV}$ with a current of $3.0 \mathrm{~mA}$. Mass spectra were acquired from $\mathrm{m} / \mathrm{z} 35$ to 780 at a rate of $30 \mathrm{spectra} / \mathrm{s}$. Following a solvent delay of $170 \mathrm{~s}$, the detector voltage was set to $-1650 \mathrm{~V}$.

\subsubsection{Data Acquisition and Processing}

The retention index of each peak was calculated by comparing the retention time of the peak with that of the alkane series C8 to C40. The compounds were identified by the comparison of the mass spectrum and retention indices of all the detected compounds with their reference standards and database in the National Institute of Standards and Technology Library 2.0 (2008) and the NEW Wiley 9 mass spectra library database. The relative quantitative peak areas of each detected peak were normalized to [13C2]-myristic acid, and the data were arranged on a two-dimensional matrix consisting of arbitrary sample names (observations) and peak area (variables). The original data were transformed into CDF format (NetCDF) using the Agilent GC/MS 5975 Data Analysis software and processed using the XCMS software (v.1.36.0; www.bioconductor.org). The processes of nonlinear retention time alignment, baseline filtration, peak identification, matching, and integration were included. Metabolites analysis and preprocessing were accomplished by SmartNuclide (Suzhou, China).

\subsubsection{Univariate, Multivariate, and Pathway Analysis}

All statistical analyses of metabolites were done based on different function modules of a powerful tool, which is available online (https://www.metaboanalyst.ca) [45]. Peaks are aligned across all samples based on their mass tolerance and retention time tolerance with the default value of $0.25(\mathrm{~m} / \mathrm{z})$ and 5 (s), respectively. Features with $>50 \%$ missing values were removed and the missing values of the remained features were replaced by a very small value (half of the minimum positive value found in the data set). No missing values were detected with the criterion. The data then underwent logarithmic transformation and normalization of auto-scaling. Fold change analysis and $t$-test were conducted to determine the Fold Change and statistical significance of each metabolite from the serum samples of piglets intravenously infused with SB compared with Con and were further used to select differential metabolites. PCA and PLS-DA were employed to picture the overall difference between the SB and the Con groups and to explore the differential metabolites. The metabolites with variable importance projection (VIP) values above 1.0, FDR (false discovery rate) $<0.1$ and Fold Change $>1.5$ or $<0.66$ were selected as differential metabolites. The differential metabolites were used to execute an enrichment analysis to explore the main pathway changed by the infusion of SB. Time-series + one experimental factor module was used to determine the time-dependent effect, SB infusion effect, and their interaction effect. Venn diagrams were generated by an online tool named Bioinformatics \& Evolutionary Genomics (http://bioinformatics.psb.ugent.be/webtools/Venn/).

\subsection{Data Analysis}

Difference in serum biochemical parameters and the relative concentrations of differential metabolites were analyzed by two-way ANOVA using PROC MIXED with time and SB treatment as the main factors in SAS, and an independent sample $t$-test (two-tailed test) was used to compare the data of differential features between two groups at each timepoint (release 8.4; SAS Institute Inc., Cary, NC, USA). Differences with a $p$-value below 0.05 were considered significant.

\section{Conclusion}

In conclusion, intravenous infusion of SB has profound effects on the body metabolism mainly including amino acid metabolism, lipid-related metabolism, and the TCA cycle. Parenteral SB may induce more amino acids to synthesize proteins and affect fat metabolism through increasing fat mobilization and decreasing the biosynthesis of stearates. 
Supplementary Materials: The following are available online at http://www.mdpi.com/2218-1989/10/1/20/s1, Table S1: Relative standard deviation (RSD) of the retention time of internal standard in QC. Table S2: Metabolites in the serum of growing pigs identified by gas chromatograph-mass spectrometry analysis. Table S3: Differential metabolites between the Con group and SB group within the whole period. Table S4: Differential metabolites between the Con group and SB group at $0 \mathrm{~min}$. Table S5: Differential metabolites between Con group and SB group at 30 min. Table S6: Differential metabolites between Con group and SB group at 60 min. Table S7: Differential metabolites between Con group and SB group at $120 \mathrm{~min}$.

Author Contributions: Y.S. and W.Z. conceived and designed the experiments; H.W., E.R., X.X., and Y.S. performed the experiments and analyzed the data; H.W. and Y.S. wrote the paper. All authors have read and agreed to the published version of the manuscript.

Funding: The research has received funding from the National Key R\&D Program of China (2018YFD0500404), and the National Natural Science Foundation of China (31572414 and 31872362).

Conflicts of Interest: The authors declare no conflict of interest.

\section{References}

1. Fu, X.; Liu, Z.; Zhu, C.; Mou, H.; Kong, Q. Nondigestible carbohydrates, butyrate, and butyrate-producing bacteria. Crit. Rev. Food Sci. Nutr. 2019, 59 (Suppl. S1), S130-S152. [CrossRef]

2. Hu, J.; Lin, S.; Zheng, B.; Cheung, P.C. Short-chain fatty acids in control of energy metabolism. Crit. Rev. Food Sci. Nutr. 2018, 58, 1243-1249. [CrossRef]

3. Wang, C.C.; Wu, H.; Lin, F.H.; Gong, R.; Xie, F.; Peng, Y.; Hu, C.H. Sodium butyrate enhances intestinal integrity, inhibits mast cell activation, inflammatory mediator production and JNK signaling pathway in weaned pigs. Innate Immun. 2018, 24, 40-46. [CrossRef]

4. Vieira, E.L.; Leonel, A.J.; Sad, A.P.; Beltrão, N.R.; Costa, T.F.; Ferreira, T.M.; Alvarez-Leite, J.I. Oral administration of sodium butyrate attenuates inflammation and mucosal lesion in experimental acute ulcerative colitis. J. Nutr. Biochem. 2012, 23, 430-436. [CrossRef]

5. Huang, C.; Song, P.; Fan, P.; Hou, C.; Thacker, P.; Ma, X. Dietary sodium butyrate decreases postweaning diarrhea by modulating intestinal permeability and changing the bacterial communities in weaned piglets. J. Nutr. 2015, 145, 2774-2780. [CrossRef]

6. Xu, J.; Chen, X.; Yu, S.; Su, Y.; Zhu, W. Effects of early intervention with sodium butyrate on gut microbiota and the expression of inflammatory cytokines in neonatal piglets. PLoS ONE 2016, 11, e0162461. [CrossRef] [PubMed]

7. Thormar, H.; Hilmarsson, H.; Bergsson, G. Stable concentrated emulsions of the 1-monoglyceride of capric acid (monocaprin) with microbicidal activities against the food-borne bacteria Campylobacter jejuni, Salmonella spp., and Escherichia coli. Appl. Environ. Microbiol. 2006, 72, 522-526. [CrossRef] [PubMed]

8. Jang, Y.D.; Lindemann, M.D.; Monegue, H.J.; Monegue, J.S. The effect of coated sodium butyrate supplementation in sow and nursery diets on lactation performance and nursery pig growth performance. Livest. Sci. 2017, 195, 13-20. [CrossRef]

9. Feng, W.; Wu, Y.; Chen, G.; Fu, S.; Li, B.; Huang, B.; Liu, J. Sodium butyrate attenuates diarrhea in weaned piglets and promotes tight junction protein expression in colon in a GPR109A-dependent manner. Cell. Physiol. Biochem. 2018, 47, 1617-1629. [CrossRef] [PubMed]

10. He, B.; Wang, M.; Guo, H.; Jia, Y.; Yang, X.; Zhao, R. Effects of sodium butyrate supplementation on reproductive performance and colostrum composition in gilts. Animal 2016, 10, 1722-1727. [CrossRef]

11. Song, B.; Li, H.; Wu, Y.; Zhen, W.; Wang, Z.; Xia, Z.; Guo, Y. Effect of microencapsulated sodium butyrate dietary supplementation on growth performance and intestinal barrier function of broiler chickens infected with necrotic enteritis. Anim. Feed Sci. Technol. 2017, 232, 6-15. [CrossRef]

12. Ahsan, U.; Cengiz, Ö.; Raza, I.; Kuter, E.; Chacher, M.F.A.; Iqbal, Z.; Cakir, S. Sodium butyrate in chicken nutrition: The dynamics of performance, gut microbiota, gut morphology, and immunity. World's Poult. Sci. J. 2016, 72, 265-275. [CrossRef]

13. Tian, L.; Zhou, X.Q.; Jiang, W.D.; Liu, Y.; Wu, P.; Jiang, J.; Xie, F. Sodium butyrate improved intestinal immune function associated with NF- $\mathrm{KB}$ and $\mathrm{p} 38 \mathrm{MAPK}$ signalling pathways in young grass carp (Ctenopharyngodon idella). Fish Shellfish Immunol. 2017, 66, 548-563. [CrossRef] 
14. Liu, M.; Guo, W.; Wu, F.; Qu, Q.; Tan, Q.; Gong, W. Dietary supplementation of sodium butyrate may benefit growth performance and intestinal function in juvenile grass carp (Ctenopharyngodon idellus). Aquac. Res. 2017, 48, 4102-4111. [CrossRef]

15. Qiu, Y.; Ma, X.; Yang, X.; Wang, L.; Jiang, Z. Effect of sodium butyrate on cell proliferation and cell cycle in porcine intestinal epithelial (IPEC-J2) cells. In Vitro Cell. Dev. Biol. Anim. 2017, 53, 304-311. [CrossRef]

16. Miletta, M.C.; Petkovic, V.; Eblé, A.; Ammann, R.A.; Flück, C.E.; Mullis, P.E. Butyrate increases intracellular calcium levels and enhances growth hormone release from rat anterior pituitary cells via the G-protein-coupled receptors GPR41 and 43. PLoS ONE 2014, 9, e107388. [CrossRef]

17. Davie, J.R. Inhibition of histone deacetylase activity by butyrate. J. Nutr. 2003, 133, 2485S-2493S. [CrossRef]

18. Walsh, M.E.; Bhattacharya, A.; Sataranatarajan, K.; Qaisar, R.; Sloane, L.; Rahman, M.M.; Van Remmen, H. The histone deacetylase inhibitor butyrate improves metabolism and reduces muscle atrophy during aging. Aging Cell 2015, 14, 957-970. [CrossRef]

19. Hamer, H.M.; Jonkers, D.M.A.E.; Venema, K.; Vanhoutvin, S.A.L.W.; Troost, F.J.; Brummer, R.J. The role of butyrate on colonic function. Aliment. Pharmacol. Ther. 2008, 27, 104-119. [CrossRef]

20. Li, G.; Yao, W.; Jiang, H. Short-Chain Fatty Acids Stimulate Porcine Adipocyte Differentiation Probably through Inhibition of Histone Deacetylases; Endocrine Society: Washington, DC, USA, 2014; Volume 35.

21. Lu, C.; Thompson, C.B. Metabolic regulation of epigenetics. Cell Metab. 2012, 16, 9-17. [CrossRef]

22. Xiong, Y.; Miyamoto, N.; Shibata, K.; Valasek, M.A.; Motoike, T.; Kedzierski, R.M.; Yanagisawa, M. Short-chain fatty acids stimulate leptin production in adipocytes through the $\mathrm{G}$ protein-coupled receptor GPR41. Proc. Natl. Acad. Sci. USA 2004, 101, 1045-1050. [CrossRef] [PubMed]

23. Katoh, K.; Tsuda, T. Effects of intravenous injection of butyrate on the exocrine pancreatic secretion in guinea pigs. J. Comp. Physiol. Psychol. 1987, 87, 569-572. [CrossRef]

24. Bartholome, A.L.; Albin, D.M.; Baker, D.H.; Holst, J.J.; Tappenden, K.A. Supplementation of total parenteral nutrition with butyrate acutely increases structural aspects of intestinal adaptation after an $80 \%$ jejunoileal resection in neonatal piglets. JPEN-Parenter. Enter. 2004, 28, 210-222. [CrossRef] [PubMed]

25. Drozdowski, L.A.; Dixon, W.T.; McBurney, M.I.; Thomson, A.B. Short-chain fatty acids and total parenteral nutrition affect intestinal gene expression. JPEN-Parenter. Enter. 2002, 26, 145-150. [CrossRef] [PubMed]

26. Ren, E.; Chen, X.; Yu, S.; Xu, J.; Su, Y.; Zhu, W. Transcriptomic and metabolomic responses induced in the livers of growing pigs by a short-term intravenous infusion of sodium butyrate. Animal 2018, 12, 2318-2326. [CrossRef] [PubMed]

27. Chen, X.; Xu, J.; Su, Y.; Zhu, W.Y. Effects of intravenous infusion with sodium butyrate on colonic microbiota, intestinal development-and mucosal immune-related gene expression in normal growing pigs. Front. Microbiol. 2018, 9, 1652. [CrossRef]

28. Sakdee, J.; Poeikhampha, T.; Rakangthong, C.; Poungpong, K.; Bunchasak, C. Effect of Tributyrin Supplementation in Diet on Production Performance and Gastrointestinal Tract of Healthy Nursery Pigs. Pak. J. Nutr. 2016, 15, 954-962.

29. Grilli, E.; Tugnoli, B.; Foerster, C.J.; Piva, A. Butyrate modulates inflammatory cytokines and tight junctions components along the gut of weaned pigs. J. Anim. Sci. 2016, 94 (Suppl. S3), 433-436. [CrossRef]

30. Casanova-Higes, A.; Andrés-Barranco, S.; Mainar-Jaime, R.C. Effect of the addition of protected sodium butyrate to the feed on Salmonella spp. infection dynamics in fattening pigs. Anim. Feed Sci. Technol. 2017, 231, 12-18. [CrossRef]

31. Val-Laillet, D.; Guérin, S.; Coquery, N.; Nogret, I.; Formal, M.; Romé, V.; Malbert, C.H. Oral sodium butyrate impacts brain metabolism and hippocampal neurogenesis, with limited effects on gut anatomy and function in pigs. FASEB J. 2018, 32, 2160-2171. [CrossRef]

32. Yu, S.; Ren, E.; Xu, J.; Su, Y.; Zhu, W. Effects of early intervention with sodium butyrate on lipid metabolism-related gene expression and liver metabolite profiles in neonatal piglets. Livest. Sci. 2017, 195, 80-86. [CrossRef]

33. Rader, D.J.; Hovingh, G.K. HDL and cardiovascular disease. Lancet 2014, 384, 618-625. [CrossRef]

34. Hong, J.; Jia, Y.; Pan, S.; Jia, L.; Li, H.; Han, Z.; Zhao, R. Butyrate alleviates high fat diet-induced obesity through activation of adiponectin-mediated pathway and stimulation of mitochondrial function in the skeletal muscle of mice. Oncotarget 2016, 7, 56071. [CrossRef] [PubMed]

35. Masud, F.; Shakoori, T.A.; Khawaja, K.I.; Ali, M.; Ameer, F. Changes in body weight and lipid profile of mice treated with sodium butyrate and metformin. Pak. J. Zool. 2016, 48, 1343-1348. 
36. Yin, F.; Yu, H.; Lepp, D.; Shi, X.; Yang, X.; Hu, J.; Gong, J. Transcriptome analysis reveals regulation of gene expression for lipid catabolism in young broilers by butyrate glycerides. PLOS ONE 2016, 11, e0160751.

37. Naveenkumar, S.; Karthikeyan, N.; Narendra Babu, R.; Veeramani, P.; Sivarama Krishnani, S.; Srinivasan, G. Effect of calcium propionate and coated sodium butyrate as an alternative to antibiotic growth promoters on the serum profile of commercial broiler chicken. Int. J. Chem. Stud. 2018, 6, 36-39.

38. Pamir, N.; Hutchins, P.; Ronsein, G.; Vaisar, T.; Reardon, C.A.; Getz, G.S.; Heinecke, J.W. Proteomic analysis of HDL from inbred mouse strains implicates APOE associated with HDL in reduced cholesterol efflux capacity via the ABCA1 pathway. J. Lipid Res. 2016, 57, 246-257. [CrossRef]

39. Marcil, V.; Delvin, E.; Seidman, E.; Poitras, L.; Zoltowska, M.; Garofalo, C.; Levy, E. Modulation of lipid synthesis, apolipoprotein biogenesis, and lipoprotein assembly by butyrate. Am. J. Physiol. Gastrointest. Liver Physiol. 2002, 283, G340-G346. [CrossRef]

40. Tabuchi, Y.; Takasaki, I.; Doi, T.; Ishii, Y.; Sakai, H.; Kondo, T. Genetic networks responsive to sodium butyrate in colonic epithelial cells. FEBS Lett. 2006, 580, 3035-3041. [CrossRef]

41. Sun, S.H.; Ou, H.C.; Jang, T.H.; Lin, L.B.; Huang, H.M. Altered phospholipid metabolism in sodium butyrate-induced differentiation of C6 glioma cells. Lipids 1997, 32, 273-282. [CrossRef]

42. Mailloux, R.J.; Bériault, R.; Lemire, J.; Singh, R.; Chénier, D.R.; Hamel, R.D.; Appanna, V.D. The tricarboxylic acid cycle, an ancient metabolic network with a novel twist. PLOS ONE 2007, 2, e690. [CrossRef] [PubMed]

43. Martin, D.B.; Vagelos, P.R. The mechanism of tricarboxylic acid cycle regulation of fatty acid synthesis. J. Biol. Chem. 1962, 237, 1787-1792. [CrossRef]

44. M Astbury, S.; M Corfe, B. Uptake and metabolism of the short-chain fatty acid butyrate, a critical review of the literature. Curr. Drug Metab. 2012, 13, 815-821. [CrossRef] [PubMed]

45. Chong, J.; Soufan, O.; Li, C.; Caraus, I.; Li, S.; Bourque, G.; Xia, J. MetaboAnalyst 4.0: Towards more transparent and integrative metabolomics analysis. Nucleic Acids Res. 2018, 46, W486-W494. [CrossRef] [PubMed]

(C) 2020 by the authors. Licensee MDPI, Basel, Switzerland. This article is an open access article distributed under the terms and conditions of the Creative Commons Attribution (CC BY) license (http://creativecommons.org/licenses/by/4.0/). 